\title{
ON SUBSURFACES OF SOME RIEMANN SURFACES
}

\author{
KIKUJI MATSUMOTO
}

Introduction. In the theory of meromorphic functions, it is important to investigate the properties of covering surfaces generated by their inverse functions. For this purpose, the study of properties of a non-compact region of a Riemann surface is useful.

Recently Kuramochi has given in his paper [5] the following very interesting theorem. Let $R$ be a Riemann surface and let $R_{0}$ be a compact domain on $R$ with compact relative boundary $\partial R_{0}$. Then

Theorem. If $R$ belongs to $O_{H B}-O_{G}\left(O_{H D}-O_{G}\right.$ resp. $)$, then $R-R_{0}$ belongs to $O_{A B}\left(O_{A D}\right.$ resp. $)$.

Here we use the following notations.

$O_{G}$ : the class of Riemann surfaces which admit no Green function.

$O_{H B}\left(O_{A B}\right)$ : the class of Riemann surfaces on which there exists no nonconstant single-valued bounded harmonic (analytic) function.

$O_{H D}\left(O_{A D}\right)$ : the class of Riemann surfaces on which there exists no nonconstant single-valued harmonic (analytic) function with finite Dirichlet-integral.

Constantinescu-Cornea [1] have investigated this theorem in detail and obtained several results. Kuramochi [6] has extended this theorem again.

On the other hand, the method given by Heins [2] may be expected to contribute to the same purpose. He introduced the concept "locally of type-Bl" using the Green functions and gave many results concerning covering properties.

We shall give, in this article, simple proofs of extended Kuramochi's theorems in Constantinescu-Cornea's way and prove some properties of covering surfaces using them and Heins' method.

For simplicity, we shall call, in this article, a non-compact or compact domain $G$ on a Riemann surface $R$ a subregion on $R$ when its relative boundary $C$ with respect to $R$ consists of at most an enumerable number of analytic noncompact or compact curves which cluster nowhere in $R$. We say that $G$ belongs

Received February 9, 1959. 
to the class $\mathrm{SO}_{H B}\left(\mathrm{SO}_{H D}\right)$ if there exists no non-constant single-valued bounded (Dirichlet-bounded) harmonic function in $G$ which vanishes continuously at every point on $C$.

1. Let $R_{1}$ and $R_{2}$ be two Riemann surfaces which admit Green functions and let $f$ be a conformal mapping of $R_{1}$ into $R_{2}$. We denote by $\mathbb{G}_{R_{1}}$ and $\mathbb{G}_{R_{2}}$ Green functions of $R_{1}$ and $R_{2}$ respectively. Then holds the equality

$$
\mathbb{S}_{R_{2}}(f(p) ; q)=\sum_{f(r)=q} n(r) \mathbb{S}_{R_{1}}(p ; r)+u_{q}(p),
$$

where $n(r)$ is the multiplicity of $f$ at $r \in R_{1}$, and $u_{q}(p)$ is the greatest harmonic minorant of $\mathscr{S}_{R_{2}}(f(p) ; q)$ on $R_{1}$.

Generally, a positive harmonic function is representable uniquely by the sum of a non-negative quasi-bounded harmonic function which is defined as the limit of a monotone non-decreasing sequence of non-negative bounded harmonic functions, and a non-negative singular harmonic function which is defined as a non-negative harmonic function dominating no positive bounded harmonic function (Parreau [9]). Heins [2] proved that $u_{q}(p)$ is quasi-bounded except for a set of $g$ of capacity zero and that the quasi-bounded component of $u_{q}(p)$ is either positive on $R_{1} \times R_{2}$ or constantly zero.

According to Heins [2], we say that $f$ is of type- $\mathrm{Bl}$ if the second alternative occurs for $f$.

Now, let $R_{1}$ and $R_{2}$ be arbitrary Riemann surfaces, and let $f$ be a conformal mapping of $R_{1}$ into $R_{2}$. We shall say that $f$ is of type- $\mathrm{Bl}$ at $q \in R_{2}$ provided that there exists a simply connected Jordan region $\Omega$ satisfying: (1) $q \in \Omega \subset R_{2}$, (2) $f^{-1}(\Omega) \neq \phi$ and (3) for each component $\Delta$ of $f^{-1}(\Omega)$, the restriction $f_{\Delta}$ of $f$ to $\Delta$ is of type- $\mathrm{Bl}$ considering $f_{\Delta}$ as to be a conformal mapping of $\Delta$ into $\Omega$. We shall say that $f$ is locally of type- $\mathrm{Bl}$ if $f$ is of type- $\mathrm{Bl}$ at each point of $R_{2}$. Then, we obtain the following:

Theorem 1. Let $R_{1}$ and $R_{2}$ be arbitrary Riemann surfaces, and let $f$ be a conformal mapping of $R_{1}$ into $R_{2}$. Then, $f$ is locally of type- $\mathrm{Bl}$ if and only if, for any compact subregion $\Omega$ on $R_{2}$ (we suppose that $\Omega$ has at least one exterior point when $R_{2}$ is compact), each component of $f^{-1}(\Omega)$ belongs to $\mathrm{SO}_{H B}$.

Proof. It is evident that $f$ is locally of type- $\mathrm{Bl}$ if, for any compact subregion $\Omega$ on $R_{2}$, each component of $f^{-1}(\Omega)$ belongs to $S O_{H B}$. 
Suppose that $f$ is locally of type-Bl. Let $\Omega$ be an arbitrary compact subregion on $R_{2}$. and let $\left\{R_{2}^{i}\right\}$ be an exhaustion of $R_{2}$ with compact relative boundaries $\partial R_{2}^{i}$. As $\Omega$ is compact in $R_{2}$, there exists an integer $i_{0}$ such that $R_{2}^{i_{0}} \supset \Omega$. (When $R_{2}$ is compact, we take as $R_{2}^{i_{0}}$ a subregion on $R_{2}$ containing $\Omega$ and having at least one exterior point.) Let $\Delta$ be any component of $f^{-1}(\Omega)$ and let $\Delta^{*}$ be the component of $f^{-1}\left(R_{2}^{i_{0}}\right)$ containing $\Delta$. And we put $A=\min _{s \in \bar{Q}} \mathbb{S}_{R^{i_{0}}}(s ; q)$, where $q$ is an arbitrary point of $R_{2}$. Consider a bounded positive harmonic function $u$ on $\Delta$ vanishing continuously on $\partial \Delta$, and denote by $u^{*}$ the subharmonic function which is equal to $u$ on $\Delta$ and to zero on $\Delta^{*}-\Delta$. Without loss of generality, we can suppose that sup $u^{*} \leqq 1$. Then, we have

$$
A u^{*} \leqq \mathbb{B}_{R^{i_{2}}}\left(f_{\Delta^{*}} ; q\right)
$$

on $\Delta^{*}$. The least harmonic majorant of $A u^{*}$ on $\Delta^{*}$ is dominated by the quasibounded component of the greatest harmonic minorant of $\mathscr{S}_{R^{i} 0_{2}}\left(f_{\Delta^{*}} ; q\right)$. By Theorem 16. 1 in [2], $f_{\Delta^{*}}$ is of type-Bl considering $f_{\Delta^{*}}$ as to be a conformal mapping of $\Delta^{*}$ into $R_{2}^{i_{0}}$, and hence the quasi-bounded component of the greatest harmonic minorant of $\mathscr{S}_{R^{i n_{2}}}\left(f_{\Delta^{*}} ; q\right)$ is identically zero in $\Delta^{*}$. Consequently, we can conclude that $u \equiv 0$ and therefore we have $\Delta \in S O_{H B}$. Thus our proof is complete.

2. Let $R$ be a Riemann surface which admits a Green function, let $\mathbb{S}_{R}(p ; q)$ be the Green function on $R$ with a pole at $q \in R$ and let $p=\varphi(t)$ be the mapping which maps the universal covering surface $R^{\infty}$ of $R$ onto $|t|<1$ one-to-one conformally. Then $\mathbb{S}_{R}(\varphi(t) ; q)$ has angular limit zero a.e. on $|t|=1$. We denote by $\tilde{f}$ the set of all points on $|t|=1$ of such kind and classify $\mathfrak{F}$ into classes by the following equivalence relation. Let $t_{1}$ and $t_{2}$ be points of $\tilde{F}$. We say that $t_{1}$ and $t_{2}$ belong to the same class provided that there exists a covering transformation $T$ of $R^{\infty}$ such that $t_{2}=T^{\prime}\left(t_{1}\right)$, where $T^{\prime}$ is the linear transformation of $|t|<1$ onto itself corresponding to $T$. We call each class an ideal boundary point and call all points of $\tilde{F}$ belonging to an ideal boundary point its image. We denote by $F$ all ideal boundary points.

If the image $\mathfrak{M}$ of a subset $M$ of $F$ is measurable on $|t|=1$, we say that $M$ is measurable and call $\omega(p ; M, R)=\omega^{*}\left(\varphi^{-1}(p) ;(\mathbb{R})\right.$ the harmonic measure of $M$ with respect to $R$, where $\omega^{*}(t ; \mathfrak{M})$ is the harmonic measure of $\mathfrak{M}$ with respect to $|t|<1$. Let $M$ be a set of positive measure. According to Constantinescu- 
Cornea [1], we say that $M$ is $H B(H D)$-indivisible if, for any bounded (Dirichletbounded) harmonic function $u(p)$ on $R, u(\varphi(t))$ has the same angular limit a.e. on the image $\mathfrak{M}$ of $M$. For instance, $F$ is $H B(H D)$-indivisible if $R$ belongs to $O_{H B}-O_{\theta}\left(O_{H D}-O_{g}\right)$. It is known that if $M$ is $H B$-indivisible, then $M$ is $H D$. indivisible.

We shall consider the class $U_{H B}\left(U_{H D}\right)$ of Riemann surfaces which contain at least one $H B(H D)$-indivisible set on their ideal boundaries. Heins [3] introduced a class $O_{L}$ of Riemann surfaces, on which there exists no non-constant single-valued Lindelöfian meromorphic function. Here we say a conformal mapping of a Riemann surface $R_{1}$ into another Riemann surface $R_{2}$ is Lindelöfian if

$$
\sum_{f(r)=q} n(r) \mathbb{S}_{R_{1}}(p ; r)<+\infty
$$

for $p$ and $q$ satisfying $f(p) \neq q$. It was proved by Heins that the relation

$$
O_{H B} \subset O_{L} \subset O_{A B}
$$

holds and that, for the class of Riemann surfaces with finite genus,

$$
O_{G}=O_{H B}=O_{L}
$$

holds.

Let $R$ be a Riemann surface belonging to $U_{H B}$, let $M$ be an $H B$-indivisible set on its ideal boundary and let $f$ be a single-valued Lindelöfian meromorphic function. Then we have for $w=f(\varphi(t))$

$$
\begin{aligned}
\sum_{f(p)=w_{w}} n(w) \mathbb{S}(t ; s) & =\sum_{f(r)=w} n(r ; f)\left\{\sum_{p(s)=r} \mathbb{S}(t ; s)\right\} \\
& =\sum_{f(r)=u} n(r ; f) \mathbb{S}_{R}(\varphi(t) ; r)<+\infty,
\end{aligned}
$$

and $f(\varphi(t))$ is Lindelöfian on $|t|<1$. Hence, we see that $f(\varphi(t))$ is meromorphic of bounded type in Nevanlinna's sense in $|t|<1$ from Heins' result: A Lindelöfian meromorphic function of the unit disc is of bounded type. So $f(\varphi(t))$ has the same angular limit a.e. on the image $\mathbb{M}$ of $M$ and we can conclude that $f$ is constant by the theorem of Lusin and Priwaloff [8].

Similary we can see that there exists no non-constant single-valued meromorphic function with finite Dirichlet-integral on any Riemann surface belonging to $U_{H D}$. Thus, we have the following relations;

$$
\begin{aligned}
& O_{H B}-O_{G} \subset U_{H B} \subset O_{L}-O_{G} \subset O_{A B}-O_{G} \\
& O_{H D}-O_{G} \subset U_{H D} \subset O_{A D}-O_{G .} .
\end{aligned}
$$


3. We shall deal with some operations introduced by Kuramochi [4] and Heins [2] for the sequel. Let $G$ be a subregion on a Riemann surface $R$, let $u$ be a positive harmonic function on $R$ and let $U$ be a positive harmonic function on $G$ vanishing continuously on $\partial G$ such that there exists at least one positive superharmonic function on $R$ dominating $U$ on $G$ (we shall call such a function $U$ admissible). We denote by $I_{G}(u)$ and $E_{G}(U)$ the upper envelope of the nonnegative subharmonic functions on $\partial G$ dominated by $u$ and vanishing continuously on $\partial G$ and the lower envelope of the positive superharmonic functions on $R$ dominating $U$ on $G$, respectively. It is easily verified that $I_{G i}(u)$ and $E_{G i}(U)$ are harmonic in $G$ and in $R$ respectively, and that $I_{G}(u)$ vanishes continuously on $\partial G$.

We shall state some properties of these operations as lemmas.

Lemma 1. Operations $I_{i r}$ and $E_{f}$ have the property of linearity.

Proof. We shall give a proof only for $I_{(i}$.

For any positive number $a$, obviously the equality

$$
I_{i}(a u)=a I_{i ;}(u)
$$

holds. Let $v$ be the same one as $u$. Then

$$
I_{i i}(u)+I_{\text {(i }}(v) \leqq u+v \quad \text { on } G .
$$

Hence

$$
I_{G i}(u)+I_{r_{i}}(v) \leqq I_{f_{i}}(u+v) \leqq u+v
$$

on $G$. Consider $\max \left(I_{G}(u+v)-u, 0\right)$ on $G$. It is subharmonic in $G$, vanishes continuously on $\partial G$ and is dominated by $v$ on $G$. Hence

$$
I_{f i}(u+v)-u \leqq \max \left(I_{i i}(u+v)-u, 0\right) \leqq I_{f i}(v)
$$

and

$$
I_{f i}(u+v)-I_{f i}(v) \leqq u
$$

Hence we have

$$
I_{i i}(u+v)-I_{G i}(v) \leqq I_{G}(u) \text {, i.e. } I_{G}(u+v) \leqq I_{i i}(u)+I_{i i}(v),
$$

and therefore we can conclude that

$$
I_{i i}(u+v)=I_{i i}(u)+I_{i i}(v) .
$$

We can prove the linearity of $E_{q}$ in the similar way. 
LeMma 2. $I_{f_{i}} \cdot E_{f}$ is an identity, that is, for any admissible positive harmonic function $U$ on $G$,

$$
I_{i}\left[E_{G}(U)\right]=U .
$$

Proof. It is evident that $E_{G}(U) \geqq U$ on $G$ and we have on $G$

$$
E_{G}(U) \geqq I_{G}\left[E_{G}(U)\right] \geqq U \text {. }
$$

Hence we have

$$
E_{G}(U) \geqq E_{G}\left[I_{G}\left(E_{G}(U)\right)\right] \geqq E_{G}(U),
$$

and, by Lemma 1 ,

$$
\begin{aligned}
E_{F_{i}}\left[I_{G}\left(E_{G}(U)\right)\right] & =E_{G}\left[I_{G}\left(E_{G}(U)\right)-U+U\right] \\
& =E_{G}\left[I_{G}\left(E_{G}(U)\right)-U\right]+E_{F_{i}}(U) .
\end{aligned}
$$

Therefore

$$
E_{G}\left[I_{I_{r}}\left(E_{G}(U)\right)-U\right]=0,
$$

and we can infer that

$$
I_{G}\left[E_{G}(U)\right]=U
$$

LeMma 3. Let $v$ be a positive harmonic function on $R$. If there exists an admissible positive harmonic function $U$ on $G$ such that $v$ is dominated by $E_{i}(U)$, then we can find an admissible function $V$ on $G$ such that

$$
v=E_{G}(V) \text {. }
$$

Proof. From $v \leqq E_{G}(U)$, we have

$$
U=I_{G}\left[E_{G i}(U)\right]=I_{G i}\left[\left(E_{G i}(U)-v\right)+v\right]=I_{G}\left[E_{G}(U)-v\right]+I_{G_{i}}(v) .
$$

Hence we have

$$
E_{G}\left[I_{f_{i}}(v)\right]+E_{f_{r}}\left[I_{G_{i}}\left(E_{f_{i}}(U)-v\right)\right]=E_{G}(U) .
$$

On the other hand, obviously

$$
E_{G}\left[I_{F_{i}}(v)\right] \leqq v \text { and } E_{f i}\left[I_{f_{i}}\left(E_{f_{i}}(U)-v\right)\right] \leqq E_{f_{i}}(U)-v \text {, }
$$

and we can conclude that

$$
v=E_{f}\left[I_{f}(v)\right] .
$$

Putting $V=I_{f i}(v)$, we see that $V$ satisfies the conditions of the lemma.

Lemma 4. Let $U$ and $U_{i}(i=1,2, \ldots)$ be admissible positive harmonic 
functions on $G$ and let $u$ and $u_{i}(i=1,2, \ldots)$ be positive harmonic functions on R. If $U=\sum_{i=1}^{\infty} U_{i}$ exists, then

$$
E_{G}(U)=\sum_{i=1}^{\infty} E_{G}\left(U_{i}\right)
$$

If $u=\sum_{i=1}^{\infty} u_{i}$ exists, then

$$
I_{G}(u)=\sum_{i=1}^{\infty} I_{G}\left(u_{i}\right)
$$

Proof. For any integer $n, U \geqq \sum_{i=1}^{n} U_{i}$ and $u \geqq \sum_{i=1}^{n} u_{i}$. Hence we have

and

$$
E_{G}(U) \geqq E_{G}\left(\sum_{i=1}^{n} U_{i}\right)=\sum_{i=1}^{n} E_{G}\left(U_{i}\right)
$$

$$
I_{G}(u) \geqq I_{G}\left(\sum_{i=1}^{n} u_{i}\right)=\sum_{i=1}^{n} I_{G}\left(u_{i}\right)
$$

Therefore

$$
E_{G}(U) \geqq \sum_{i=1}^{\infty} E_{G}\left(U_{i}\right) \text { and } I_{G}(u) \geqq \sum_{i=1}^{\infty} I_{G}\left(u_{i}\right)
$$

By Lemma 3, we can find a positive harmonic function $V$ on $G$ vanishing continuously on $\partial G$ such that $E_{G}(U) \geqq E_{G}(V)=\sum_{i=1}^{\infty} E_{G}\left(U_{i}\right)$. Hence, for any integer n,

$$
U=I_{G}\left[E_{G}(U)\right] \geqq V=I_{G}\left[E_{G}(V)\right] \geqq I_{G}\left[\sum_{i=1}^{n} E_{G}\left(U_{i}\right)\right]=\sum_{i=1}^{n} U_{i} .
$$

Hence we can see that $U=V$ and therefore

$$
E_{G}(U)=E_{G}(V)=\sum_{i=1}^{\infty} E_{G}\left(U_{i}\right)
$$

Next we shall prove the latter equality. If we take an arbitrary point $p$ on $R$, then we can find an integer $n$ for given positive number $\varepsilon$ such that $\sum_{i=n+1}^{\infty} u_{i}(p)<\varepsilon$. From $I_{G}\left(\sum_{i=n+1}^{\infty} u\right)(p) \leqq \sum_{i=n+1}^{\infty} u_{i}(p)<\varepsilon$, we have

$$
I_{G}(u)(p)-\varepsilon \leqq\left(\sum_{i=1}^{\infty} I_{I_{i}}\left(u_{i}\right)\right)(p) \leqq\left(\sum_{i=1}^{\infty} I_{G}\left(u_{i}\right)\right)(p) .
$$

Since we can take $\varepsilon$ as small as we please and $p$ is an arbitrary point on $R$, we have

$$
I_{(i}(u) \leqq \sum_{i=1}^{\infty} I_{G}\left(u_{i}\right)
$$

and hence 


$$
I_{G}(u)=\sum_{i=1}^{\infty} I_{G}\left(u_{i}\right)
$$

We shall say that a positive harmonic function $u$ is minimal if, for any positive harmonic function $v$ dominated by $u$, there exists a constant $c(0<c \leqq 1)$ such that $v=c u$. Then we obtain the following lemma.

Lемма 5. Let $u$ be a positive minimal harmonic function on $R$. If $I_{G}(u)$ is positive, then $I_{G}(u)$ is also minimal on $G$.

Proof. Let $U$ be a positive harmonic function on $G$ dominated by $I_{G}(u)$. Then $U$ vanishes continuously on $\partial G$. We have

$$
E_{G}(U) \leqq E_{G}\left[I_{G}(u)\right] \leqq u,
$$

and on account of the minimality of $u$ we can find a constant $c(0<c \leqq 1)$ such that

$$
E_{G}(U)=c u \text {. }
$$

Hence

$$
U=I_{G}\left[E_{G}(U)\right]=c I_{G}(u) .
$$

Let $H D$ be the class of non-negative harmonic functions, each of which is the limiting function of a monotone non-increasing sequence of positive harmonic functions with finite Dirichlet-integrals. We shall say that a positive harmonic function $u$ belonging to $H D$ is minimal in $H D$ if, for any positive member $v$ of $H D$ dominated by $u$, there exists a constant $c(0<c \leqq 1)$ such that $v=c u$.

Constantinescu and Cornea [1] proved that if $u$ and $v$ belong to $H D$, the greatest harmonic minorant $u \wedge v$ of the superharmonic function $\min (u, v)$ and the least harmonic majorant $u \vee v$ of the subharmonic function $\max (u, v)$ also belong to $H D$.

Lemma 6. Let $u$ be a positive HD-minimal harmonic function on $R$, and let $G$ be a subregion not belonging to $S_{H D}$. If there exists an admissible positive harmonic function $U$ on $G$ having a finite Dirichlet-integral such that $E_{G}(U)$ dominates $u$ on $R$, then $I_{F}(u)$ is also minimal in HD on $G$.

Proof. By Lemma 3 we can see that there exists an admissible function $V$ on $G$ such that $E_{G}(V)=u$, because $E_{G}(U) \geqq u$. Hence $U \geqq V$ and $u \geqq u \wedge U \geqq V$ on $G$. Obviously $u \wedge U$ vanishes continuously on $\partial G$. We see that $u \wedge U=V$ because $V$ is the upper envelope of positive subharmonic functions dominated 
by $u$ and vanishing continuously on $\partial G$. Therefore $V$ belongs to $H D$.

If $W$ is a positive harmonic function on $G$ belonging to $H D$ and dominated by $V$, then $E_{G}(W)$ also belongs to $H D$ on $R$ and $E_{s}(W)=c u$ for some constant $c(0<c \leqq 1)$. In fact, let $\left\{W_{i}\right\}$ be a monotone non-increasing sequence of harmonic functions with finite Dirichlet-integrals having $W$ as their limiting function. Then the sequence $\left\{U \wedge W_{i}\right\}$ also has $W$ as their limiting function. It is seen that $E_{G}\left(U \wedge W_{i}\right) \in H D$ and $\lim _{i \rightarrow \infty} E_{G}\left(U \wedge W_{i}\right)=E_{G}(W) \leqq E_{G}(V)=u$. Since $u$ is minimal in $H D$ on $R$, there exists a constant $c$ such that $E_{G}(W)=c u$.

Hence we have $W=I_{G}\left[E_{G}(W)\right]=c I_{G}(u)=c V$. Thus we can conclude that $I_{G}(u)$ is minimal in $H D$ on $G$.

If $M$ is a $H D$-indivisible set such that, for any $H D$-indivisible set $M^{\prime}$ containing $M$, the harmonic measure of $M^{\prime}-M$ with respect to $R$ is zero, then we call $M$ a maximal $H D$-indivisible set. Constantinescu-Cornea [1] proved that $M$ is $H B$ (maximal $H D$ ) -indivisible if and only if the harmonic measure $\omega(p ; M)$ of $M$ with respect to $R$ is minimal (minimal in $H D$ ). For the problem when subregions on a Riemann surface belonging to $U_{H B}$ or $U_{H D}$ belong to $U_{H B}$ or $U_{H D}$, Lemmas 5 and 6 with this result give some answers.

The condition of the last lemma is equivalent to the condition "frei" given by Constantinescu-Cornea [1].

4. According to Constantinescu and Cornea [1], we denote by $O_{H B_{n}}\left(O_{H D_{n}}\right)$ $(1 \leqq n \leqq \infty)$ the class of Riemann surfaces, the ideal boundary of which is null or consists of at most $n H B$ (maximal $H D$ )-indivisible sets. These classes are the same ones considered by Kuramochi [6]. In fact, as Constantinescu and Cornea proved, $O_{H B_{n}}\left(O_{H D_{n}}\right)(1 \leqq n<\infty)$ coincides with the class of Riemann surfaces on which there exist at most $n$ number of linearly independent bounded (Dirichlet-bounded) harmonic functions. We note that $O_{H B_{1}}=O_{H B}$ and $O_{H n_{1}}$ $=O_{H D}$.

Now, we give proofs of Kuramochi's Theorems [5], [6].

TheOREM 2. (Kuramochi) If a Riemann surface $R$ belongs to $O_{H B_{n}}-O_{G}$ $(1 \leqq n \leqq \infty)$ and a subregion $G$ on $R$ does not belong to $\mathrm{SO}_{H B}$, then $G$ belongs to $O_{L}$.

Proof. Suppose that the ideal boundary of $R$ consists of just $m(\leqq n)$ number of $H B$-indivisible sets $M_{i}(i=1,2, \ldots, m)$. Let $\omega_{i}(i=1,2, \ldots, m)$ 
be the harmonic measure of $M_{i}$ in $R$. Then each $\omega_{i}$ is minimal and $\sum_{i=1}^{m} \omega_{i} \equiv 1$. Since $G$ does not belong to $S O_{H B}, I_{G} 1=\sum_{i=1}^{m} I_{G}\left(\omega_{i}\right)$ is positive. Consequently for some $i_{0}, I_{G}\left(\omega_{i_{0}}\right)$ is positive and minimal on $G$ by Lemma 5 .

We map the universal covering surface $G^{\infty}$ of $G$ onto $|t|<1$, and denote the mapping function by $p=\varphi(t)$. Let $M$ be the set on $|t|=1$ such that $I_{G}\left(\omega_{i_{0}}\right) \circ \varphi$ has angular limit 1 a.e. on it and 0 a.e. on $(|t|=1)-M$. Then $M$ is of measure positive and on account of the minimulity of $I_{G}\left(\omega_{i_{0}}\right), M$ is an $H B$-indivisible set. Hence the region $G$ belongs to $U_{H B}$ and by the relation $\left(^{*}\right.$ ) we can see that $G \in O_{L}$. Thus the proof is complete.

Kuroda [7] introduced a class $O_{A B}^{0}$ of Riemann surfaces, on every subregion of which there exists no non-constant single-valued bounded analytic function with a real part vanishing continuously on its relative boundary. He proved that each Riemann surface belonging to $O_{A B}^{0}$ has Iversen property and gave the relation

$$
O_{H B} \subset O_{A B}^{0} \subset O_{A B}
$$

and for the class of Riemann surfaces with finite genus,

$$
O_{G}=O_{H B} \subset O_{A B}^{\circ} \subseteq O_{A B} .
$$

The subregion $G$ of Theorem 2 obviously does not belong to $O_{A B}^{0}$, because there exist non-constant single-valued meromorphic functions on $G$ not having Iversen property. Hence we have

$$
O_{L} \text { 韦 } O_{A B}^{0} \text {. }
$$

Further, $O_{H D}$ is not a subclass of $O_{L}$ in virtue of Tôki's example [10] and we obtain

$$
O_{L} \text { 事 } Q_{H D} \text {. }
$$

Theorem 3. (Kuramochi) If a Riemann surface $R$ belongs to $O_{H D_{n}}-O_{G}$ $(1 \leqq n \leqq \infty)$ and a subregion $G$ on $R$ does not belong to $S_{H D}$, then $G$ belongs to $O_{A D}$.

Proof. Suppose that the ideal boundary of $R$ consists of just $m(\leqq n)$ number of maximal $H D$-indivisible sets $M_{i}(i=1,2, \ldots, m)$. Let $\omega_{i}(i=1,2$, $\ldots, m$ ) be the harmonic measure of $M_{i}$ with respect to $R$. Then $\omega_{i}$ belongs to $H D$ and is minimal in $H D$ (cf. [1]). Since $G$ does not belong to $S O_{H n}$ and 
since $S O_{H D}=S O_{H B D}$, there exists a positive bounded harmonic function $U$ having a finite. Dirichlet-integral and vanishing continuously on $\partial G$. By Dirichlet principle we see that $E_{G}(U)$ has also a finite Dirichlet-integral and $E_{G}(U)=\sum_{i=1}^{m} \alpha_{i} \omega_{i}$. Since $E_{G}(U)$ is positive, for some $i_{0}, \alpha_{i_{0}}$ is positive and $\frac{1}{\alpha_{i_{0}}} E_{G}(U)=E_{G}\left(\frac{1}{\alpha_{i_{0}}} U\right)$ $\geqq \omega_{i_{0}}$. Hence by Lemma 6 , we can conclude that $I_{G}\left(\omega_{i_{0}}\right)$ is minimal in $H D$ on $G$.

We map the universal covering surface $G^{\infty}$ of $G$ onto $|t|<1$ by $\varphi$ and denote by $M$ the set on $|t|=1$ such that $I_{G}\left(\omega_{i_{0}}\right) \circ \varphi$ has angular limit 1 a.e. on $M$ and 0 a.e. on $(|t|=1)-M$. It is seen that $M$ is of positive measure and is maximal $H D$-indivisible because of the $H D$-minimality of $I_{G}\left(\omega_{i_{0}}\right)$ (cf. [1]). Hence $G \in U_{H D}$ and by the relation $\left(^{*}\right)$ we can see that $G \in O_{A D}$. Thus our theorem is proved.

5. In this section we shall state some results which are deduced from Theorems 1 and 2 .

THEOREM 4. If a Riemann surface $R$ belongs to $O_{H B_{n}}(1 \leqq n \leqq \infty)$, then any non-constant single-valued meromorphic function $f$ on $R$ is locally of type-B1.

Proof. Let $\Omega$ be an arbitrary subregion on the $w$-plane having at least one exterior point. Then all components of $f^{-1}(\Omega)$ belong to $S O_{H_{B}}$ by Theorem 2. Thus we can see that $f$ is locally of type-Bl by Theorem 1 .

Corollary. Let $R$ be a Riemann surfuce belonging to $O_{H_{B_{n}}}(1 \leqq n \leqq \infty)$, and let $\Phi$ be the covering surface of the w-plane generated by a non-constant single-valued meromorphic function $f$ on $R$. Then every connected piece $\emptyset_{\Delta}$ of $\emptyset$ on any disc $\Delta$ in the $w$-plane covers each point of $\Delta$ the same number of times except for at most an $F_{\sigma}$-set of capacity zero.

Proof. This corollary is immediate from Theorem 4 and Theorem 21.2 in $[2]$.

THEOREM 5. Let $R$ be a Riemann surface belonging to $O_{H_{B}}(1 \leqq n \leqq \propto)$ and let $G$ be a subregion on $R$ not belonging to $\mathrm{SO}_{H B}$. Then the cluster set of any non-constant single-valued meromorphic function $f$ on $G$ at the ideal boundary of $G$ is the whole w-plane, and the range of values of $f$ contains all values of the w-plane except for at most an $F_{\mathrm{o}}$-set of capacity zero.

Proof. Without loss of generality, we may suppose that $f$ is analytic on 
aG. By Theorem 2, $G$ belongs to $O_{L}$ and $f$ is not Lindelöfian. Heins proved in [3] that if, for some $p_{0} \in G, \sum_{f(r)=w} n(r) \mathscr{G}_{G}\left(p_{0}, r\right)<+\infty$ for a set of $w$ of positive capacity, then $f$ is Lindelöfian on $G$. Hence $f$ takes each value infinitely often except for an $F_{\sigma}$-set of capacity zero.

6. Here we shall be concerned with the subsurfaces on Riemann surfaces of the class $O_{H D_{n}}$.

THEOREм 6. Let $f$ be a non-constant single-valued meromorphic function on a Riemann surface $R$. If there exist a point $w_{0}, n-1(n<\infty)$ number of subregions $c_{i}$ and a sequence of Jordan regions $\Omega_{i}$ of the w-plane such that $c_{i} \cap c_{j}$ $=\phi$ for $i \neq j, w_{\theta} \notin \bigcup_{i=1}^{n-1} \bar{c}_{i}, \Omega_{i} \supset \bar{\Omega}_{i+1}$ and $\bigcap_{i=1}^{\infty} \Omega_{i}=w_{0}$, and that, for each $i$, at least one component $\delta_{i}$ of $f^{-1}\left(c_{i}\right)$ and one component $\Delta_{i}$ of $f^{-1}\left(\Omega_{i}\right)$ do not belong to SOHD, then $R$ does not belong to $O_{H D_{n}}{ }^{1)}$

To prove this theorem, we give the following:

THEOREM 7. Let $R$ be a Riemann surface. Then $R$ does not belong to $O_{H B_{n}}$ $\left(O_{H D_{n}}\right.$ resp. $)(n<\infty)$ if there exist $n+1$ subregions $G_{i}(i=0,1,2, \ldots, n)$ disjoint from each other on $R$ such that $G_{i} \notin S O_{H B}$ for all $i\left(G_{0} \notin S O_{H B}\right.$ and $G_{i} \notin S O_{H D}$ for $i=1,2, \ldots, n$ resp.).

Proof. Suppose that $R$ belongs to $O_{H B_{n}}\left(O_{H D_{n}}\right)$. Then the boundary of $R$ consists of just $m(\leqq n)$ number of $H B$ (maximal $H D$ )-indivisible sets $M_{k}$ $(k=1,2, \ldots, m)$. Since $G_{i} \notin S O_{H B}\left(S O_{H D}\right) \quad(i=1,2, \ldots, n)$, we can find for each $i \neq 0$ in the same way as in the proofs of Theorems 2 and 3 a harmonic measure $\omega_{k}(p)=\omega\left(p ; M_{k}\right)$ of $M_{k}$ such that $I_{G_{2}}\left(\omega_{k}\right)>0$. Furthermore we can see that $I_{G_{j}}\left(\omega_{k}\right)=0$ for $j=0, \ldots, i-1, i+1, \ldots, n$. In fact, for $i \neq j$,

$$
E_{G_{i}} I_{\sigma_{i}}\left(\omega_{k}\right)+E_{G_{j}} I_{G_{j}}\left(\omega_{k}\right) \leqq \omega_{k},
$$

and from the minimality of $\omega_{k}$ and the fact that $\sup _{G_{i}} I_{G_{i}}\left(\omega_{k}\right)=1$

$$
E_{G i} I_{G i}\left(\omega_{k}\right)=\omega_{k} \text {. }
$$

Hence we have $E_{G_{j}} I_{G_{j}}\left(\omega_{k}\right)=0$ and $I_{G_{j}}\left(\omega_{k}\right)=I_{G_{j}} E_{G_{j}} I_{G_{j}}\left(\omega_{k}\right)=0$. Thus we can see that, for any $\omega_{k}, I_{F_{0}}\left(\omega_{k}\right)=0$ and $I_{G_{0}}(1)=I_{F_{0}}\left(\sum_{k=1}^{m} \omega_{k}\right)=\sum_{k=1}^{m} I_{G_{0}}\left(\omega_{k}\right)=0$. This contradicts the condition: $G_{0} \notin S O_{H B}$, which proves the theorem.

1) The auther proved only the case $n=1$ and the extension of the present form is due. to Kuroda. 
Proof of Theorem 6. By Theorem $1, f$ is not locally of type- $\mathrm{Bl}$, so by Theorem 17.1 in [2] the set of points $w$ in any closed neighbourhood of $w_{0}$, at which $f$ is not of type- $\mathrm{Bl}$, is of positive capacity. Let $w_{1} \neq w_{0}$ be such a point, satisfying $w_{1} \notin \bigcup_{i=1}^{n-1} \bar{c}_{i}$, then for some $i, \Omega_{i}$ does not contain $w_{1}$ and $\Omega_{i} \cap\left(\bigcup_{i=1}^{n-1} c_{i}\right)=\phi$. Choosing a positive number $\rho$ satisfying that $\left(\Omega_{i} \cup\left(\bigcup_{i=1}^{n-1} c_{i}\right)\right)$ $\cap\left(\left|w-w_{1}\right|<\rho\right)=\phi$, we can find among components of $f^{-1}\left(\left|w-w_{1}\right|<\rho\right)$, a component $\Delta_{0}$ not belonging to $\mathrm{SO}_{H B}$ and satisfying $\Delta_{0} \cap \Delta_{i}=\phi$ and $\Delta_{0} \cap \delta_{i}=\phi$. By Theorem 7, $R$ does not belong to $O_{H D_{n}}$.

TheOREM 8. Let $R$ be a Riemann surface belonging to $O_{H D_{n}}(1 \leqq n \leqq \infty)$, let $\Phi$ be the covering surface of the w-plane generated by a non-constant singlevalued meromorphic function $f$ on $R$, and let $\Phi_{\rho}$ be a connected piece of $\emptyset$ on $\left|w-w_{0}\right|<\rho$. If the area of $\Phi_{p}$ is finite, then the restriction $f_{\rho}$ of $f$ to the component $\Delta_{p}$ of $f^{-1}\left(\left|w-w_{0}\right|<\rho\right)$ corresponding to $\Phi_{p}$ is of type- $\mathrm{Bl}$ of $\Delta_{p}$. Hence $\Phi_{p}$ covers each point of $\left|w-w_{0}\right|<\rho$ the same number of times except for at most a closed set of capacity zero, and $\Phi_{\mu}$ is finitely sheeted.

Proof. Suppose that $f_{p}$ is not of type-Bl. Then, by Theorem 1 , there exists a positive number $\rho_{0}<\rho$ such that a component $\Delta_{\rho_{0}}$ of $f^{-1}\left(\left|w-w_{0}\right|<\rho_{0}\right)$ exists and does not belong to $\mathrm{SO}_{H F}$. Let $\omega$ be the harmonic measure of $\left|w-w_{0}\right|$ $=\rho_{0}$ with respect to the ring domain $\left(\rho_{0}<\left|w-w_{0}\right|<\rho\right)$, and let $\omega^{*}$ be the superharmonic function such that $\omega^{*}$ is equal to $\omega$ on $\rho_{0}<\left|w-w_{0}\right|<\rho$ and to 1 on $\left|w-w_{0}\right| \leqq \rho_{0}$. Put $A=\max \left|\operatorname{grad} \omega^{*}\right|$. Then $A$ is finite and $D\left(\omega^{*} \circ f\right)$ $\leqq A^{2} D\left(f_{\mathrm{p}}\right)=A^{2} \times$ (the area of $\left.\Phi_{\mathrm{p}}\right)<+\infty$. Hence, by Dirichlet principle, the greatest harmonic minorant $u$ of $\omega^{*} \circ f$ of $\Delta_{p}$ has a finite Dirichlet-integral. Since $\Delta_{\rho_{0}}$ does not belong to $\mathrm{SO}_{H B}$, there exists a positive bounded harmonic function $u_{0}$ such that $u_{0}=0$ on $\partial \Delta_{\rho_{0}}$ and sup $u_{0}=1$. Denote by $u_{0}^{*}$ the subharmonic function such that $u_{0}^{*}=u_{0}$ on $\Delta_{\rho_{0}}$ and $u_{0}^{*}=0$ on $\Delta_{\rho}-\Delta_{\rho_{\theta}}$, then $\left.u_{0}^{*} \leqq \omega\right)^{*} \circ f_{\rho}$, $0<E u_{0}^{*} \leqq \omega^{*} \circ f$ because of superharmonicity of $\omega^{*} \circ f$, and we can conclude that $0<E u_{0}^{*} \leqq u$ and $\Delta_{\rho}$ does not belong to $S O_{H \rho}$. This contradicts Theorem 3 . Thus our theorem is established.

It is evident that this theorem implies Kuramochi's result (Theorem 12 in $[6])$. 


\section{REFERENCES}

[1] C. Constantinescu and A. Cornea : Über den idealen Rand und einige seiner Anwendungen bei Klassifikation der Riemannschen Flächen, Nagoya Math. J. 13 (1958), 166233.

[2] M. Heins: On the Lindelöfian principle, Ann. of Math. 61 (1955), 440-473.

[ 3 ] M. Heins : Lindelofian maps, Ann. of Math. 62 (1955), 418-445.

[4] Z. Kuramochi : Relations between harmonic dimensions, Proc. Japan Acad. 30 (1954), 576-580.

[5] Z. Kuramochi: On the behaviour of analytic functions on abstract Riemann surfaces, Osaka Math. J. 7 (1955), 109-127.

[6] Z. Kuramochi : On the ideal boundaries of abstract Riemann surfaces, Osaka Math. J. 10 (1958), 83-102.

[7] T. Kuroda: On analytic functions on some Riemann surfaces, Nagoya Math. J. 10 (1956), 27-50.

[8] N. Lusin et I. Priwaloff : Sur l'unicite et la multiplicité des fonctions analytiques, Ann. Sci. Ecole Norm. Sup. 3 Série, 42 (1925), 143-191.

[9] M. Parreau: Sur les moyennes des fonctions harmoniques et analytiques et la classification des surfaces de Riemann, Thèse, Paris, 1952.

[10] Y. Tòki: On the examples in the classification of open Riemann surfaces (I), Osaka Math. J. 5 (1953), 267-280.

\section{Mathematical Institute}

Hiroshima University 